\title{
159 QUALITY OF LIFE AMONG END-OF-LIFE FORMER COMMERCIAL PLASMA DONORS INFECTED WITH HIV IN RURAL HENAN, CHINA
}

Y Sheng, ${ }^{1} \mathrm{Z} \mathrm{Qiu}_{1}{ }^{2}$ Y He, ${ }^{3} \mathrm{Y}$ Zhang ${ }^{4}{ }^{1}$ School of Nursing of Peking Union Medical College; ${ }^{2}$ Department of Society of Beijing University; ${ }^{3}$ Henan Provincial Department of Public Health; ${ }^{4}$ School of Nursing of Tian Jin University

10.1136/bmjspcare-2013-000491.159

Background China has began providing antiretroviral therapy to people living with HIV/AIDS (PLWHA) since 2003.

Aim Purpose of present study was to investigate quality of life and happiness, as well as their needs in hospice care at their end-of-life among HIV-positive people in rural Henan.

Methods One-hundred AIDS patients at their end-of -life were selected from Weishi, Zhenping and Tanghe counties, Henan province of middle China using convenience sampling. WHO Quality of Life for HIV (WHOQOL-HIV) BREF Chinese version was used to measure their quality of life, and Memorial University of Newfoundland Scale of Happiness (MUNSH) was used to measure their subjective welfare. Personal in-depth interview and focus group discussion were used to learn their needs for hospice care at end-of-life of the patients.

Results Overall quality of life was moderate among the patients with an average score of 12.62 and SD of 1.97 , higher in domains of spirituality, religion and personal belief (with an average score of $14.40 \pm 2.96$ ) and psychological domain (13.58 \pm 2.06$)$, and lower in independence $(12.15 \pm 2.15)$ and environment (12.50 \pm 3.28 ) domains. Average MUNSH score was 21.00 \pm 6.20 , with moderate range.

Discussion and Conclusion Palliative care model used was beneficial to the patients. In-depth interviews indicated that Henan provincial government's policy of treatment and care for AIDS patients has had a beneficial impact on them at their end-of-life overall, although care component could be improved further by assisting their families as a whole. 